\title{
Philippe Bourdin e Gerard Loubinoux ed., Les Arts de la Scène et la Révolution française
}

\section{Paola Perazzolo}

\section{(2) OpenEdition}

1 Journals

\section{Edizione digitale}

URL: http://journals.openedition.org/studifrancesi/34733

DOI: 10.4000/studifrancesi.34733

ISSN: 2421-5856

\section{Editore}

Rosenberg \& Sellier

\section{Edizione cartacea}

Data di pubblicazione: 1 novembre 2005

Paginazione: 420-422

ISSN: 0039-2944

Notizia bibliografica digitale

Paola Perazzolo, «Philippe Bourdin e Gerard Loubinoux ed., Les Arts de la Scène et la Révolution

française», Studi Francesi [Online], 146 (XLIX | II) | 2005, online dal 30 novembre 2015, consultato il 18 avril 2021. URL: http://journals.openedition.org/studifrancesi/34733; DOI: https://doi.org/10.4000/ studifrancesi.34733 


\title{
Philippe Bourdin e Gerard Loubinoux ed., Les Arts de la Scène et la Révolution française
}

\author{
Paola Perazzolo
}

\section{NOTIZIA}

PHILIPPE BOURDIN e GERARD LOUBINOUX ed., Les Arts de la Scène et la Révolution française, Grenoble, Presses Universitaires Blaise-Pascal, 2004, pp. 606.

1 Il presente volume riunisce numerosi interventi riguardanti gli "Arts de la Scène» (teatro, opera, danza) durante la Rivoluzione e l'Impero. Sintesi delle tre giornate di studio tenutesi a Grenoble rispettivamente nel maggio 1998, nel settembre 1999 e nell'aprile 2001, quest'opera testimonia del fascino che la nozione di «scène bâtarde» da intendersi come «lieu de croisement des répertoires et d'affranchissement des cadres académiques» (p. 9) - da lungo tempo esercita sugli autori, che hanno giustamente valutato il periodo rivoluzionario come «crucial et déterminant» da questo punto di vista. Fenomeno socio-letterario unico, risultato di una «rivoluzione» artistica legata alla rimessa in discussione di strutture precedenti e soffocanti così come all'improvviso confronto con una libertà creativa ed espressiva senza precedenti, la produzione degli «Arts de la Scène» rivoluzionari si impone per ricchezza e complessità, soprattutto se esaminata come rapporto tra arte e Rivoluzione. Rapporto strettissimo oltre che importante per la comprensione di un decennio in cui tutto si fa teatro, in cui la politica orienta il teatro, non solo a livello di scrittura, ma anche di repertorio e di messa in scena. I mutamenti socio-politici, l'attualità storica, la riorganizzazione istituzionale dell'universo teatrale - cfr. il decreto sulla libertà dei teatri, la soppressione e la successiva riammissione della censura, l'obbligo delle rappresentazioni "par et pour le peuple» -, la velocità di metaformosi di repertori e influenze rendono la produzione rivoluzionaria unica per ricchezza, impatto sociale, terni e innovazioni, generiche e non. È questa complessità e ricchezza che le 
comunicazioni del presente volume rendono appieno, esaminando i rapporti tra arte e società da molteplici e diversi punti di vista, offrendo un lavoro non "completo» compito impossibile considerato il periodo in esame e l'approccio pluridisciplinare adottato - ma sicuramente importante.

2 La prima sezione «De la loi à la scène: les institutions parisiennes» delinea i mutamenti del panorama teatrale durante il decennio rivoluzionario. I primi due studi vertono sulla libertà dei teatri e su alcune sue conseguenze: in Le débat sur la liberté des théâtres en France, 1789-1790 (pp. 39-54), Gregory S. BRown tratteggia la crisi della ComédieFrançaise e l'aggravarsi della situazione dei comédiens, rievocando uno dei dibattiti pubblici più sentiti all'epoca, mentre Michèle SAJOUS D'ORIA, Un nouveau droit: construire des théâtres (pp. 55-72) riflette sull'architettura delle nuove e numerosissime sale edificate o ristrutturate dopo il 1791, chiedendosi se e quanto i nuovi teatri siano effettivamente «rivoluzionari» nella concezione dello spazio e della disposizione dei posti. David TRoTt, Le théâtre de foire à l'époque révolutionnaire: rupture ou continuité? (pp. 73-92), evidenzia come durante il decennio il poco istituzionale "théatre de foire» si trasformi senza tuttavia svanire: nonostante l'aspirazione ufficiale a fare del palcoscenico una «école de vertu» e la progressiva scomparsa di troupes e rappresentazioni, il théatre de foire sopravvive come pratica scenica, come esprit forain. Alessandro DI PROFIO, Les Bouffons de la reine. L'opéra italien à Paris sous la Révolution (pp. 93-116) rievoca le vicende dell'opéra italien in Francia prima dell'apertura del Théâtre de Monsieur nel 1789 e fino all'agosto 1792. L'autore sottolinea come questo teatro, un «pure produit de l'Ancien Régime» (p.115) quanto a organizzazione, repertorio e pubblico, sia stato «rivoluzionario» solo in quanto ha contribuito a definire una tripartizione nell'organizzazione degli spettacoli lirici prima inesistente - opera, opera comica e opera italiana - che permarrà per tutto il secolo successivo. Termina questa prima sezione la comunicazione di David challou, L'Opéra de Paris sous le Consulat et l'Empire (pp. 117-130), che rammenta la strumentalizzazione napoleonica delle scene e del prestigio dell'Opéra, strumentalizzazione basata sulla reintegrazione di un monopolio e di privilegi tipici dell'Ancien Régime.

3 La seconda sezione, "Troupes et salles en province et à l'étranger» ha il merito di offrire una panoramica su repertori, teatri e troupes non parigini. In Artistes et missions patriotiques en Rhône-et-Loire (1793-1794) (pp. 131-162), Philippe BouRDIN spiega come a Lione l'esperienza scenica di alcuni attori patrioti sia stata reinvestita nella produzione di immagini e cerimonie - soprattutto di decristianizzazione - a beneficio del Comitato di salute pubblica. Gli studi di Clothilde TREHOREL, Le théâtre de Dijon: artistes et spectacles entre 1789 et 1810 (pp. 163-180), e di Karine LARGE, Les théâtres de Nantes sous la Révolution française (pp. 189-204), pongono invece l'accento sulla moltiplicazione delle compagnie amatoriali a Digione e a Nantes e sulla conseguente politicizzazione del repertorio, mentre Christine LE BOZEC, Le théâtre à Rouen pendant la Révolution française (pp. 181-188), rammenta le vicissitudini teatrali di Rouen. La città, dotata di un grande teatro affidato anche a direttori prestigiosi tra cui Neuville e Mme de Montansier -, costituisce un vero e proprio caso: ad eccezione di Parigi, nessun'altra municipalità riuscirà ad organizzare su cosi vasta scala rappresentazioni «par et pour le peuple». Concludono questa sezione Cyril tRIOLAIRE, Le théâtre dans le Puy-de-Dôme sous le Consulat et l'Empire (pp. 205-234), che tratteggia la vita teatrale a Clermont-Ferrand e a Riom durante il dominio napoleonico, e Karine RANCE, Le théâtre français de Hambourg (pp. 235-250), che 
sottolinea la funzione sociale ed episodicamente catartica delle rappresentazioni del teatro francese creato nel 1794 ad Amburgo.

4 Sei comunicazioni costituiscono la terza sezione, «Pouvoir et répertoire». L'interessante intervento di Michel BIARD, Le Pour et le Contre, ou la critique théâtrale parisienne face aux ruptures de 1791 (pp. 251-272), esamina le posizioni di vari giornali in un anno cruciale qual è il 1791. Durante i primi mesi, l'argomentazione politica verte soprattutto sulla ricezione di pièces anticlericali e sulla rappresentazione della figura di Luigi XVI dopo la fuga del monarca, mentre successivamente l'inasprirsi della situazione politica condurrà alla progressiva scomparsa di posizioni filomonarchiche. Jacqueline RAZGONNIKOFF, Théâtre de la Nation: les aléas du répertoire, de la prise de la Bastille à la fermeture (14 juillet 1789-3 septembre 1793). Du patriotisme à la réaction (pp. 273-292), traccia la storia della Comédie-Française attraverso l'esame del suo repertorio. Se i comédiens mettono in scena anche novità o opere di circostanza, come il celebre Charles IX di Chénier, il loro repertorio resta prevalentemente classico e comico, ben poco «révolutionnaire», il che condurrà alla chiusura del teatro e all'incarcerazione della troupe. Brutus à la scène: autour du décret du 2 août 1793 (pp. 293-312), di Antoinette et Jean EHRARD, con la collaborazione di F. EHRARD, mette in dubbio la reale efficacia del decreto dell'agosto 1793, espressione delle convinzioni di una minoranza piuttosto che del grande pubblico. Ciò premesso, cercando nei repertori dei teatri Molière, de la Nation e de la République la presenza, personale o «métaphorique» (p. 293) di Brutus, gli autori mostrano come la figura dell'eroe romano domini comunque la scena rivoluzionaria, a livello sia testuale che di richiamo intertestuale. Seguono due comunicazioni sull'opposizione silenziosa al potere. In Présence d'Arlequin sous Robespierre (pp. 313-326), Martin NADEAU spiega come il carattere orale e improvvisato dei canovacci, per loro natura refrattari alla censura, il tema delle rappresentazioni di Arlecchino e, in definitiva, la stessa esistenza pubblica della maschera si oppongano alla politica culturale dell'anno II, volta alla «régénération de la nature humaine», diventando così atti di «politique vécue» (p. 325), mentre Françoise LE BORGNE, Le drame de la Révolution dans Le Drame de la vie de Restif de la Bretonne (pp. 327-338), sottolinea come in quest'opera l'apparente rifiuto di una presa di posizione celi in realtà una critica implicita dei miti e delle esaltazioni rivoluzionarie proposti dalle numerose pièces patriottiche coeve. Patrick ТАIEB, in La réunion des théâtres Favart et Feydeau en 1801 et l'«opéra comique révolutionnaire» (pp. 339-368), espone i cambiamenti operati dall'estetica rivoluzionaria sul genere, ambiguo ma di grande successo, dell'»opéra comique». La consapevolezza dell'autorità collettiva e delle possibilità propagandistiche del palcoscenico portano infatti a una supremazia delle parole sulla musica, caratterizzata da una grande semplicità e dall'onnipresenza nell'orchestra di strumenti a fiato e degli ottoni, caratteristiche riconducibili alla pratica delle feste patriottiche.

5 La quarta sezione, «La part de l'imaginaire», propone comunicazioni molto interessanti su temi e figure ricorrenti nelle rappresentazioni teatrali, intese come diretta espressione dell'immaginario e delle preoccupazioni collettive. Marie-Laurence NETTER, Imagination, imaginaire et convention dans le théâtre de la Révolution française (pp. 369-380), si interroga sulla convenzionalità tematica di numerose opere politiche dell'epoca, che oppongono in modo caricaturale e superficiale l'ecclesiastico cupido, l'affarista, l'aristocratico arrogante alla semplicità e alle virtù patriottiche, concludendo che queste stesse convenzioni, per quanto o proprio in quanto ripetute, sono «porteuses de 
l'imaginaire même de la Révolution, qu'elles représentent la Révolution revée» (p. 380), mentre Erica Joy MANNuccI, Le militaire dans le théâtre de la Révolution française (pp. 381-394), mostra come la rievocazione di avvenimenti militari, frequente nelle pièces d'attualità, assuma a volte toni e accenti personali e più intimi, pur mantenendosi patriottica. In L'imaginaire scénique de la prison sous la Révolution. Eloquence et plasticité d'un lieu commun (pp. 395-418), Olivier B ARA esamina l'evoluzione della rappresentazione scenica della prigione, già presente nel teatro classico ma ora diversamente interpretata. Dal 1789 al 1791 la prigione diventa un tema d'attualità: le pièces evocano esplicitamente la Bastiglia, esempi di incarcerazione civile quali l'affaire Calas o, più indirettamente, realtà conventuali, in cui le segrete, luoghi di costrizione presenti ma sempre celati, sono suggerite più che mostrate. Più tardi, il tema delle segrete, pur obbedendo al gusto per il gotico, si mescolerà ancora al tema politico: dopo il 9 Termidoro la prigione richiamerà inevitabilmente il Terrore. Gérard Loubinoux, in Les figures du théâtre de Joseph Aude (pp. 419-438), esamina le opere composte da Aude tra il 1789 e il 1804, ritenendolo un autore rappresentativo di questo periodo poiché la sua produzione si pone al limite tra teatro politico e divertissement. La lunga comunicazione di Françoise DARTOIS, Les spectacles dansés pendant la Révolution et l'Empire (pp. 439-486) prende in considerazione i circa sessanta balletti rappresentati all'Opéra tra il $1793 \mathrm{e}$ il 1799. Se la maggior parte di essi riprende terni mitologici o classici, è possibile notare come la Rivoluzione raggiunga anche l'aristocratica arte della danza, considerata come un «outil de propagande introduisant une représentation du peuple en mouvement signifiant ses engagements idéologiques» (p. 477) e in quanta tale aperta a soggetti contemporanei e alla messa in scena di parate militari.

6 L'ultima sezione del volume concerne le «Postérités du théâtre révolutionnaire». Le Portique républicain et les enjeux de la mobilisation des arts autour de brumaire an VIII (pp. 487-508) di Jean-Luc CHAPPEY delinea l'azione repubblicana svolta dal Portique tra il 1799 e il 1800 per mobilitare tutte la forme d'acte al servizio della Repubblica, a riaffermare lo «statuto» dell'arte e la missione repubblicana dell'artista, finché l'avvenimento di un nuovo regime e di una nuova concezione del pubblico non ne rese la posizione impossibile e anacronistica. In Jean Anouilh, Romain Rolland et l'écriture de la Révolution (pp. 509-524), Patrick BERTHIER ritrova nel fascino esercitato dalla figura di Robespierre un legame tra la produzione di due autori antitetici quali Anouilh e Rolland, mentre in Révolutionnaires, Jean Dasté, Jean Vilar? (pp. 525-544), Michel BOURGUIGNON offre un aperçu personale della carriera e delle personalità di Jean Dasté e di Jean Vilar, sottolineando come entrambi realizzino un «véritable modèle politique hérité des Lumières» (p. 543) in quanto fautori di un teatro «enseignant» e sociale, rivolto ad un pubblico popolare e non necessariamente parigino. Per concludere, Irène TIEDER, Marat/Sade. Dramaturgie et scénographie (pp. 545-554), esamina l'opera di Peter Weiss, concepita come un immaginario confronto tra due personaggi così opposti come Marat e Sade, e spiega come alcuni degli interrogativi sollevati - il dubbio sull'efficacia dell'azione politica, la riflessione sul ruolo dell'individuo nella società e sulla discordanza tra idealismo e realtà rivoluzionaria -, tipici degli anni sessanta a cui risale la pièce riprendano anche alcuni grandi interrogativi del decennio rivoluzionario.

7 A partire dai primi anni del Bicentenario, gli studi sull'epoca e sulla letteratura rivoluzionarie si sono notevolmente arricchiti, rendendo giustizia ad una produzione che in precedenza era stata ingiustamente penalizzata. Ma molto resta ancora da fare, a causa - grazie? - alla ricchezza e alla complessità del periodo considerato. In 
quest'ottica, e anche grazie all'eterogeneità e all'approccio pluridisciplinare delle comunicazioni, il presente volume costituisce uno strumento utilissimo per la conoscenza o l'approfondimento della vita teatrale del periodo rivoluzionario, giustamente ricondotta ai suoi rapporti e legami con il potere e la società che la influenzano e la dirigono. A dimostrazione, se ce ne fosse bisogno, che «Arte» e «Rivoluzione» non sono termini antitetici. 